\title{
Source Analysis Of Auditory Steady-State Responses In Acoustic And Electric Hearing
}

\author{
Robert Luke*1, Astrid De Vos ${ }^{* 1,2}$, and Jan Wouters ${ }^{1}$ \\ ${ }^{1}$ Research Group Experimental ORL, Department of Neurosciences, \\ KU Leuven - University of Leuven, Belgium \\ ${ }^{2}$ Parenting and Special Education Research Unit, Faculty of \\ Psychology and Educational Sciences, KU Leuven - University of \\ Leuven, Belgium
}

\begin{abstract}
Speech is a complex signal containing a broad variety of acoustic information. For accurate speech reception, the listener must perceive modulations over a range of envelope frequencies. Perception of these modulations is particularly important for cochlear implant (CI) users, as all commercial devices use envelope coding strategies. Prolonged deafness affects the auditory pathway. However, little is known of how cochlear implantation affects the neural processing of modulated stimuli. This study investigates and contrasts the neural processing of envelope rate modulated signals in acoustic and CI listeners.

Auditory steady-state responses (ASSRs) are used to study the neural processing of amplitude modulated (AM) signals. A beamforming technique is applied to determine the increase in neural activity relative to a control condition, with particular attention paid to defining the accuracy and precision of this technique relative to other tomographies. In a cohort of 44 acoustic listeners, the location, activity and hemispheric lateralisation of ASSRs is characterised while systematically varying the modulation rate $(4,10,20,40$ and $80 \mathrm{~Hz})$ and stimulation ear (right, left and bilateral). We demonstrate a complex pattern of laterality depending on both modulation rate and stimulation ear that is consistent with, and extends, existing literature.

We present a novel extension to the beamforming method which facilitates source analysis of electrically evoked auditory steady-state responses (EASSRs), In a cohort of 5 right implanted unilateral CI users, the neural activity is determined for the $40 \mathrm{~Hz}$ rate and compared to the acoustic cohort. Results indicate that $\mathrm{CI}$ users activate typical thalamic locations for $40 \mathrm{~Hz}$ stimuli. However, complementary to studies of transient stimuli, the CI population has atypical hemispheric laterality, preferentially activating the contralateral hemisphere.
\end{abstract}

*Authors contributed equally. 


\section{Introduction}

Speech is a complex signal containing a broad variety of acoustic information. For accurate speech perception, the temporal envelope of speech is particularly important (Shannon et al. 1995). The temporal envelope consists of variations in the overall amplitude of the sound pressure wave, characterised by rates of modulation between 2 and $50 \mathrm{~Hz}$ (Rosen 1992). More specifically, modulation rates around 4 and $20 \mathrm{~Hz}$ are considered fundamental for speech perception due to their relation with the rate of syllables and phonemes, important phonological segments in speech (Edwards and Chang 2013). Envelope coding is used to convey information to CIs users, further accentuating the importance of modulation sensitivity for this population $(\mathrm{Fu} 2002$, Wouters et al. 2015).

Given the importance of envelope rate modulations in speech, it is of particular interest to understand where and how these modulations are represented and processed within the human auditory pathway. The capability of the auditory system to process temporal modulations can be investigated by means of ASSRs. ASSRs are neural responses evoked during auditory stimulation with a temporally modulated stimulus, and reflect how well the auditory system phase locks to the stimulus rhythm (Picton et al. 2003). These evoked potentials have been linked to several perceptual outcomes, ASSRs are used clinically to objectively determine frequency specific hearing thresholds (Rance et al. 1995), and have also been related to speech perception (Dimitrijevic et al. 2004, Poelmans et al. 2012a). In CI users, EASSRs have been used to predict hearing thresholds (Hofmann and Wouters 2012) and related to modulation sensitivity (Luke et al. 2015).

A variety of imaging methods have shown ASSRs activate the entire auditory pathway, with different modulation rates preferentially activating different pathway segments (Rance 2008). Higher modulation rates $(\gtrsim 80 \mathrm{~Hz})$ predominantly activate the brainstem, with lower rates $(\lesssim 40 \mathrm{~Hz})$ activating the thalamus and auditory cortices (Reyes et al. 2004, Roß et al. 2000, Herdman et al. 2002, Giraud et al. 2000, Langers et al. 2005). Less is known about the hemispheric lateralisation of modulated auditory stimuli, where changes may represent variation in underlying functional relationships between different processing centres (Langers et al. 2005). A right hemispheric preference has been observed for $40 \mathrm{~Hz}$ ASSRs regardless of stimulation ear (Roß et al. 2000). $4 \mathrm{~Hz}$ modulated signals exhibit contralateral auditory cortex (AC) activation for monaural stimulation (Langers et al. 2005), and right activation 
during bilateral stimulation (Hymers et al. 2010, Prendergast et al. 2010). However, scalp level analysis indicates there may be additional richness to this variation that is yet to be quantified on a source level (Poelmans et al. 2012a, Gransier et al. 2016). We hypothesise that source analysis of ASSRs will reveal hemispheric lateralisation that is dependant on modulation rate and stimulation modality.

Typical development of the auditory pathway is presumably beneficial for speech perception, and is facilitated by providing auditory stimulation within developmental sensitive periods (Kral and Sharma 2012). Unfortunately, deprivation of auditory stimulation is accompanied by modifications to the auditory system (Lazard et al. 2011) and reduced performance (Lazard et al. 2012, Kral and Sharma 2012). The effect of reintroducing auditory stimulation is less well known, investigation has been hindered by incompatibilities between CIs and magnetic resonance imaging (MRI) (Majdani et al. 2009), and has required the development of alternative source analysis methods. Gilley et al. (2008) demonstrated reorganised cortical pathways using a electroencephalography (EEG) sLORETA source reconstruction of cortical evoked potentials. Wong and Gordon (2009) introduced an EEG beamforming method (Van Veen and Buckley 1988) for analysing the sources of transient responses, this method was used to demonstrate unilateral implantation results in contralateral dominance that can be mitigated with timely bilateral implantation (Gordon et al. 2013). However, CI recipients primarily rely on envelope cues transmitted via sustained modulations (Friesen et al. 2001). We hypothesise that similar to transient stimuli, enhanced contralateral activation will be seen for modulated stimuli.

To summarise, we hypothesise that EEG beamforming will provide a measure with adequate sensitivity to reveal hemispheric lateralisation of ASSRs. This methodology will reveal hemispheric lateralisation that is modulation rate and stimulation modality dependent. Further, in the CI population we hypothesise that enhanced contralateral activation will be seen for modulated stimuli. Below we introduce the EEG beamforming method and quantify the accuracy and precision with reference to other tomographies. In the acoustically stimulated population, the location, strength and lateralisation is characterised for modulation rates $4,10,20,40$ and $80 \mathrm{~Hz}$ with stimuli presented monaurally to the left ear, monaurally to the right ear and bilaterally. This result is used as a baseline for which to compare the CI cohort. With extensions to remove the CI artifacts, the beamforming method is ap- 
plied to five right ear implanted CI participants for the $40 \mathrm{~Hz}$ rate, and the two cohorts are compared.

\section{Materials \& Methods}

\subsection{Participants}

Two groups of listeners participated in this study. The first cohort comprised of 44 acoustic hearing participants ( 5 male, mean age 22). All acoustic hearing participants had audiometric thresholds below $25 \mathrm{~dB}$ HL on all octave frequencies between 0.25 and $8 \mathrm{kHz}$ and reported no history of language or learning disabilities, brain injury or neurological disorders. The second cohort comprised of five CI, i.e. electrical, hearing participants (3 male, mean age 61). All electrical hearing participants were Cochlear Nucleus CI users with devices implanted in the right ear. Testing was approved by the Medical Ethics Committee of the UZ Leuven (approval number B322201316755 for acoustic hearing participants and B32220072126 for CI participants) and informed consent was obtained from all participants.

\subsection{Stimuli}

\subsubsection{Acoustical Stimulation}

Acoustic stimuli consisted of amplitude modulated speech-weighted noise with $100 \%$ modulation depth, presented to the participants at $70 \mathrm{~dB}$ SPL through ER-3A insert phones. The carrier noise was adopted from the 'Leuven Intelligibility Sentence Test' and represents the long-term average speech spectrum of 730 sentences of a female speaker (Van Wieringen and Wouters 2008). Five modulation rates $(4,10,20,40$ and $80 \mathrm{~Hz}$ ) were presented in three modalities (monaurally to the left ear, monaurally to the right ear and bilaterally to both ears). The broad range of modulation rates was chosen to investigate ASSRs generated along the entire auditory pathway from brainstem to $\mathrm{AC}$. Additionally, 4, 10 and $20 \mathrm{~Hz}$ rates were chosen to represent typical syllable and phoneme rates in speech Greenberg et al. 1996. Greenberg and Takayuki 2004). The 40 and $80 \mathrm{~Hz}$ rates were selected as they produce the largest scalp signal-to-noise ratios (SNRs) and are the most commonly studied rates (Picton et al. 2003). Both uni- and bilateral stimulation modalities were included, for it has been shown to significantly affect lateralisation on source (Langers et al. 2005) and scalp level (Poelmans et al. 2012a). A silence condition was also measured as a reference for the 
beamforming procedure. Each of the sixteen conditions was presented for 5 minutes in a randomised order.

\subsubsection{Electrical Stimulation}

Electrical stimulation consisted of amplitude modulated biphasic pulse trains presented via the NIC research platform (Hofmann and Wouters 2010, 2012). All stimuli were presented in bipolar mode, both the active and return CI electrodes were located within the cochlea. Bipolar mode was used as it induces shorter EEG artifacts than in monopolar mode, and which can be removed on the ipsilateral side. In each participant three CI electrode pairs were stimulated, a basal, middle and apical electrode pair. Ten min of EEG were recorded per CI electrode pair. A pulse rate of 900 pulses per second (pps) was used, to match the users daily clinical processor. Stimuli were modulated between the threshold and comfort level as described in Luke et al. (2015). A $40 \mathrm{~Hz}$ modulation rate was used as it has the highest SNR and can be consistently detected in the CI population (Gransier et al. 2016, Luke et al. 2015, Deprez et al. 2017), and laterality results have been established in the acoustic population (Roß et al. 2003).

\subsection{EEG Acquistion}

EEGs were recorded with a Biosemi ActiveTwo system using sixty-four active $\mathrm{Ag} / \mathrm{AgCl}$ electrodes mounted in head caps according to the 10-20 electrode system. The use of electrode caps ensures consistent electrode spacing and positioning. All recordings were administered in a double-walled soundproof booth with a Faraday cage. Participants were asked to relax while watching a soundless movie. Watching a movie prevents the participants from falling asleep and ensures non-attentive listening to the auditory stimuli, thereby controlling for possible effects of sleep and attention (Roß et al. 2003). To avoid excessive movement or muscle tensions, participants were encouraged to lie quietly and relax during auditory stimulation. The data was sampled at a rate of $8192 \mathrm{~Hz}$ and saved for offline processing.

\subsection{Source Analysis}

\subsubsection{Beamforming}

Beamforming was used to determine the increase in neural activity relative to the silence condition. This method was inspired by Wong and Gordon (2009), but applied in the frequency domain (Groß et al. 2001). Electrodes 
at the back of the head (P9, P10, P07, PO8, P7, P8, O1, O2, Oz and Iz) often had a poor connection to the scalp, especially in participants with longer hair, and were removed from all participants to ensure consistent analysis. Recorded signals were referenced to the average before high pass filtering from $2 \mathrm{~Hz}$. Epochs of $1.024 \mathrm{~s}$ were extracted, and $10 \%$ of these were rejected based on the peak to peak value. The Cross Power Spectral Density (CPSD) at the modulation rate was calculated using the methods provided by Gramfort et al. (2013, 2014), and used to calculate the Neural Activity Index (NAI) as described by Van Veen and Buckley (1988). The NAI may be interpreted as an estimate of the source to noise variance (Van Veen et al. 1997), a value of one indicates the source variance is the same in the control and stimulus condition, a value higher than one indicates increased activity in the stimulus condition relative to the control condition, a value less than one indicates reduced activity in the stimulus condition relative to the control condition.

For modulation rates $40 \mathrm{~Hz}$ and below, bilateral correlated generators were expected. Correlated sources are known to cause errors in beamforming techniques. To mitigate these errors, a bilateral region suppression technique was employed Dalal et al. (2006). Additionally, subspace projection was used to improve the SNR (Sekihara et al. 2001). An automated procedure was used to detect dipoles. The automated procedure detected local peaks in the NAI, and placed dipoles at these locations (Lemire 2006). No restrictions were placed on the number of identified dipoles, figure 2 illustrates that the number of dipoles was low and representative of the raw data. A realistic finite element model was used for the leadfield [BESA GmbH]. To validate the source analysis procedure, analysis was performed at frequencies off the modulation rate, and visually inspected to ensure spurious sources weren't detected. Additionally, the accuracy and precision were investigated against locations reported from other tomographies (section 2.5.1).

\subsubsection{Resampling Analysis}

Beamforming requires a minimum SNR to accurately estimate neural activity (see section 2.5.1). To achieve this SNR, the CPSD matrices were averaged across subjects, this also mitigates other sources of error such as variation in cap placement and differences with the leadfield head model. Bootstrapping was used to determine the variability of each estimate (Efron 1979), 10000 resamples were taken with replacement to obtain a measure of variation on each estimate. For visualisation, and to observe if any one participant was 
driving the results, jackknifing was also performed on the grand average data (Tukey 1958).

\subsection{Analysis Of EEG Data Elicited By Acoustical Stimula- tion}

\subsubsection{Location Accuracy}

The accuracy of the imaging technique was investigated by comparing the location of extracted dipoles to previously reported ASSR locations. The accuracy was determined for the $40 \mathrm{~Hz}$ modulation rate, as this is the only modulation rate where locations have been reported using different methods. The average location reported by Reyes et al. (2004), Steinmann and Gutschalk (2011), Reyes et al. (2005), and Teale et al. (2008) was used as a reference location. The location difference was defined as the euclidean distance between the determined location and the mean reference location. Bootstrapping was used to obtain multiple distance estimates. To determine a minimum required recording time, the distance to the literature location was determined for increasing effective recording times. Different effective recording times were simulated by varying the number of CPSDs included in each bootstrap sample.

\subsubsection{Description Of Neural Activity}

Estimates of dipole locations, $\overline{\mathrm{NAI}}$ and variability were calculated for each modulation rate and stimulation modality. Additionally, a measure of hemispheric laterality was calculated (Poelmans et al. 2012b, Gordon et al. 2013), The Laterality Index (LI) was defined as $\mathrm{LI}=(R-L) /(R+L)$, where $L$ and $R$ represents the [NAI of the left and right dipole respectively, a value of -1 indicates completely left lateralised activity, a value of 0 indicates balanced hemispheric activation.

\subsection{Analysis Of EEG Data Elicited By Electrical Stimulation}

The location, $\mathrm{NAI}$ and laterality were also calculated for the CI group. Due to the limited number of participants a jackknifing procedure was used instead of bootstrapping, this provided a method to determine if any single participant was driving the results (Tukey 1958). To improve the SNR, the CPSDs were averaged across CI electrode conditions. However, for illustrative purpose the dipole locations for each condition are also shown in figure $8 \mathrm{a}$ 

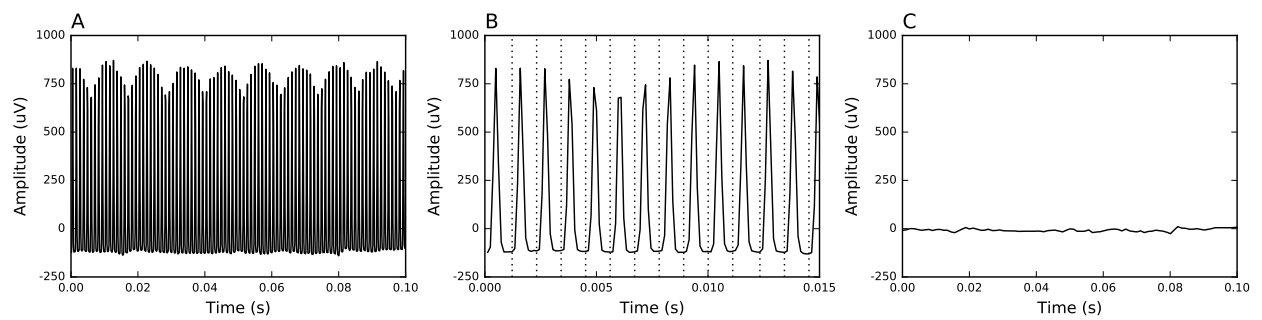

Figure 1: CI artifact removal example. A) Raw EEG measurement containing CI artifact. B) Zoomed in view of data with dashed vertical lines indicating clean samples used for blanking. C) Data after blanking and filtering.

\subsubsection{Electrical Artifact Removal}

EEG data recorded from CI participants contains artifacts from the radio frequency (RF) transmission and electrical stimulation. To mitigate the effect of these artifacts, two modifications were made to the signal processing pipeline. First, blanking was used to remove the majority of the electrical artifact (Hofmann and Wouters 2012, Deprez et al. 2017), this technique was used as the raw signal was still required (Luke and Wouters 2016). Figure 1 illustrates the $\mathrm{CI}$ artifact removal process for an example recording. A raw EEG measurement containing large CI artifacts is shown in figure 1 A, figure $1 \mathrm{~B}$ highlights the samples between each artifact which are used for interpolation in the blanking procedure. Figure $1 \mathrm{C}$ illustrates the measurement after the blanking procedure and high pass filtering, the large abrupt CI artifacts are removed from the data. Secondly, EEG electrodes touching the CI were removed from analysis prior to the rereferencing step. To ensure electrode removal did not affect laterality measures, mirror electrodes were also removed from analysis. Two frontal electrodes were also removed as they were noisy. In total, the additional removed electrodes were P5, P6, P7, P8, F5, F6.

\subsection{Comparison Of Groups}

To determine if the electrical hearing group had similar neural activation to the acoustic group, the NAI and laterality were compared. To ensure that the additional signal processing in the CI group (i.e. electrical artifact removal) did not affect the lateralisation index, the same processing was applied to the acoustic hearing data in the $40 \mathrm{~Hz}$ right ear condition. 10000 resamples were taken from the reprocessed acoustic hearing group, providing 
a distribution of the $\mathrm{LI}$ for the acoustic population. The probability that the CI LI was drawn from the acoustic distribution was then calculated.

\section{Results \& Discussion}

\subsection{Acoustic Analysis}

Figure 2 shows the raw grand average source estimates for bilateral stimulation at 40 and $80 \mathrm{~Hz}$. The maximum $\mathrm{NAI}$ is shown along each dimension, each point represents an analysed voxel, the color and size represent the NAI. Blue squares indicate individual jackknife estimates, the mean of the jackknife estimates is indicated with red markers. The reference literature location is shown for the $40 \mathrm{~Hz}$ condition in green. The $40 \mathrm{~Hz}$ condition shows two focal points of increased activity in the left and right hemisphere, these points are 12 and $8 \mathrm{~mm}$ from the left and right literature location respectively, little variation is shown across jackknife estimates. The $80 \mathrm{~Hz}$ condition shows centralised activity with increased variation relative to the $40 \mathrm{~Hz}$ condition, the increased variation is due to the lower SNR and represents a greater region of uncertainty on the estimate.

\subsubsection{Location Accuracy}

The neural generators of $40 \mathrm{~Hz}$ ASSRs have previously been reported using MRI (Langers et al. 2005), positron emission tomography (PET) (Reyes et al. 2004), magnetoencephalography (MEG) (Roß et al. 2000) and EEG (Herdman et al. 2002). Some studies predetermined the region of interest (Herdman et al. 2002), while others investigated the entire brain (Reyes et al. 2004). In this study, beamforming was used as not to limit the analysis to predetermined regions, and not place a-priori assumptions about the number of active sources. Due to the low amplitude of ASSRs and novel application of this imaging technique, particular attention was paid to quantifying the accuracy and precision of the results.

The accuracy of the beamforming imaging technique was investigated relative to an averaged literature reference location. In total 44 participants contributed $5 \mathrm{~min}$ of data per condition, totalling $220 \mathrm{~min}$ of recordings. Bootstrapping on the entire $40 \mathrm{~Hz}$ data set produced an average distance to the literature reference of 12.7 (SD 3.8) mm. This constant offset can be seen in figure $2 \mathrm{a}$ and is likely caused by differences with the headmodel. 

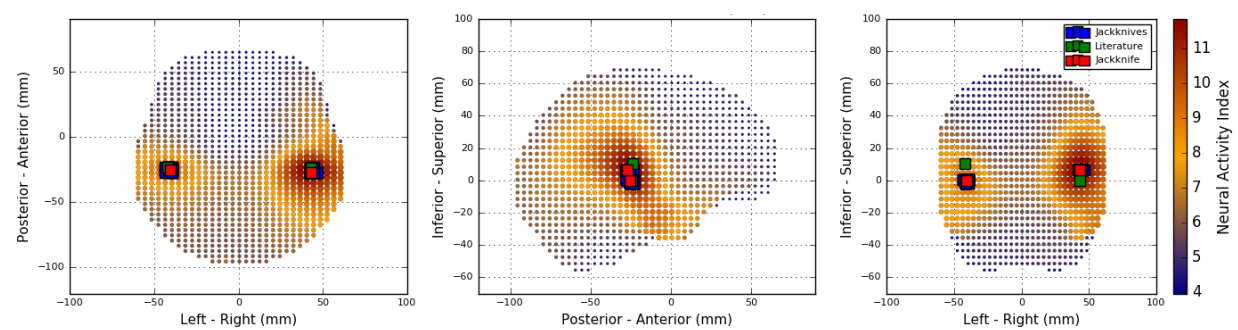

(a) $40 \mathrm{~Hz}$
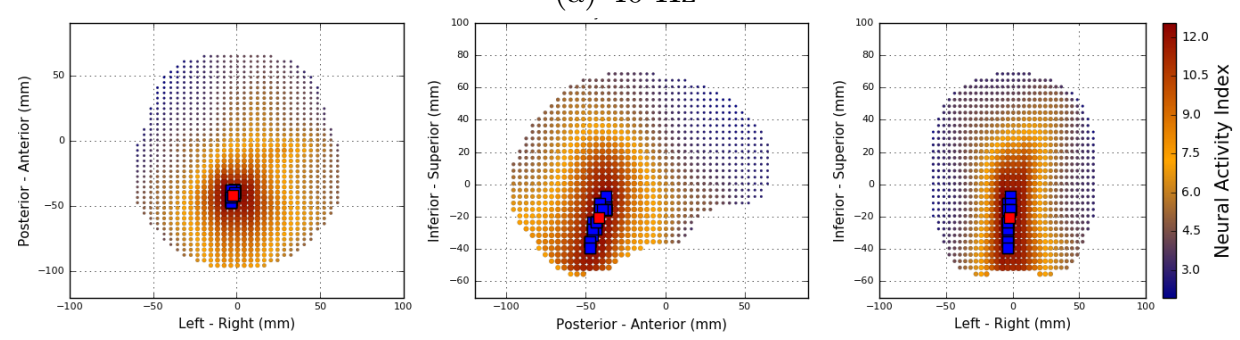

(b) $80 \mathrm{~Hz}$

Figure 2: Beamforming source estimates for 40 and $80 \mathrm{~Hz}$ bilateral stimulation in the acoustic hearing group. The maximum NAI is shown along each dimension. Each point indicates an analysed voxel. Point size and color represent the NAI. Individual jackknife estimates are shown in blue, the mean jackknife estimate is shown in red, the literature reference is overlaid in green. 


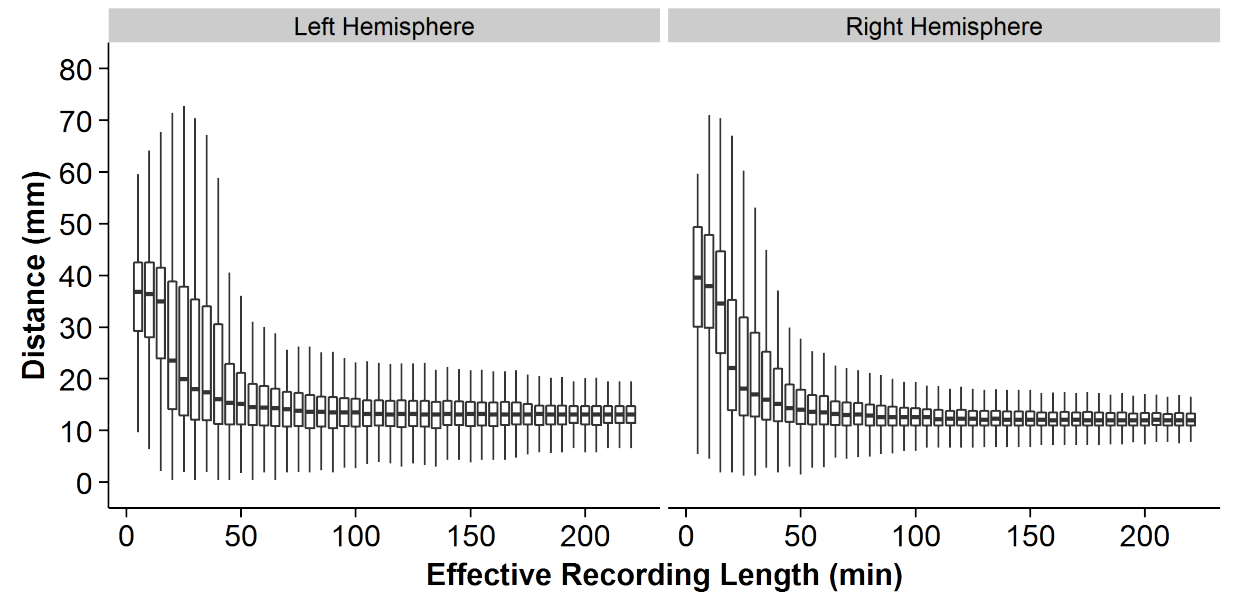

Figure 3: Source analysis accuracy for $40 \mathrm{~Hz}$ condition, combining the three stimulation modes, as a function of effective recording length. Left and right facets represent the accuracy of the left and right hemisphere respectively.

By reducing the resample size we can simulate smaller effective recording lengths, this is illustrated in figure 3 . The distance to the reference location reduces with increasing measurement time, and the location of the dipole stabilises from approximately 50 mins. Greater accuracy is observed for the right hemisphere, presumably due to the larger activation and thus increased SNR. This is the expected level of accuracy that can be obtained with EEG source analysis (Cuffin et al. 2001). Localisation improvements may be observed with improved head models (Cho et al. 2015) or advanced imaging techniques (Lucka et al. 2012, Hymers et al. $\mid$ 2010).

\subsubsection{Description Of Neural Activity}

Figure 4 shows the location estimates for all modulation rates, the mean location and standard deviation is shown for each dimension. Central sources were observed for the $80 \mathrm{~Hz}$ modulation rate, consistent with the expectation of a brainstem generator (Herdman et al. 2002, Picton et al. 2003). Thalamic activation was seen for the $40 \mathrm{~Hz}$ condition [see also section 3.1.1]. The coordinates of the $40 \mathrm{~Hz}$ sources were $(-31,-26,3) \mathrm{mm}$ and $(34,-24$, 6) $\mathrm{mm}$ for the left and right sources respectively in the standard Talairach coordinate system. For modulation rates below $40 \mathrm{~Hz}$, the area of increased NAI extended and became increasingly inferior and lateral, this is illustrated 

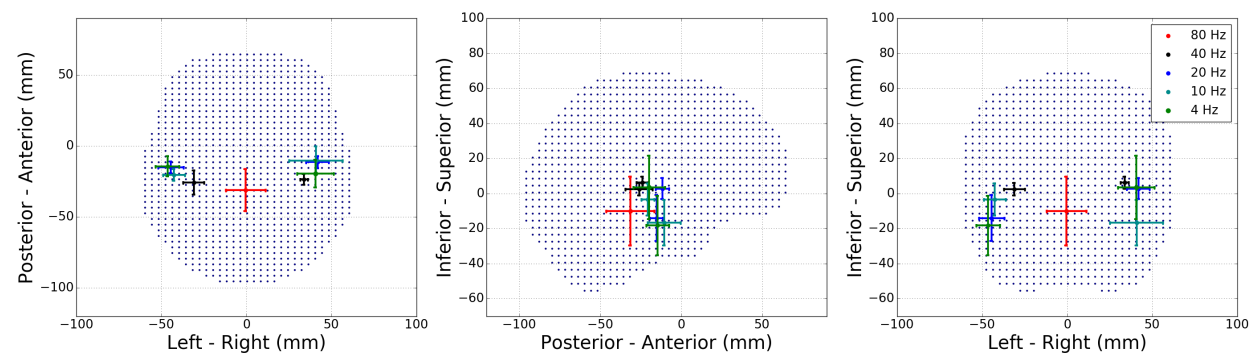

Figure 4: Source location of ASSRs for different modulation rates, averaged across modalities. Color indicates the modulation rate. Error bars indicate the standard deviation in each dimension.

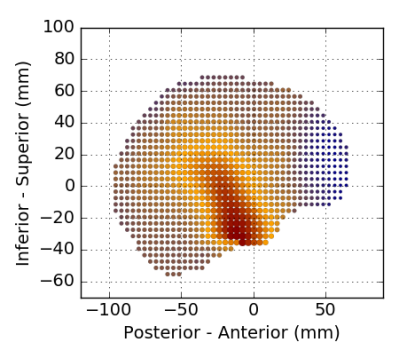

(a) $4 \mathrm{~Hz}$

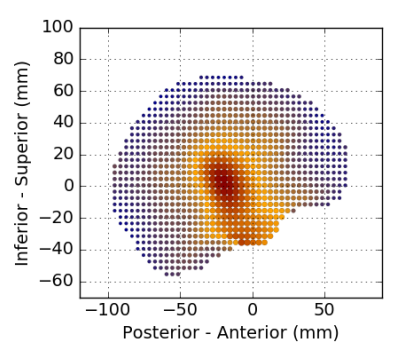

(b) $10 \mathrm{~Hz}$

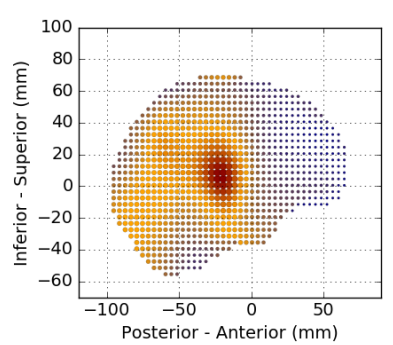

(c) $40 \mathrm{~Hz}$

Figure 5: Grand average source estimates for 4, 10 and $40 \mathrm{~Hz}$ modulation rates presented to the right ear. Activity is focused for the $40 \mathrm{~Hz}$ condition, and becomes increasingly inferior and lateral for lower modulation rates.

in figure 5. As the region of cortical activation has been shown to be essentially the same across different AM rates (Giraud et al. 2000), the smooth transition of source location below $40 \mathrm{~Hz}$ may indicate a transition from medial geniculate body (MGB) to primary and secondary ACs that has been smeared by the imaging technique. Coherent source suppression was used to mitigate the effect of multiple generators across hemispheres (Dalal et al. 2006), but cannot be used within a small area due to the low spatial resolution of EEG. For low modulation rates, increased care should be taken when interpreting a single dipole fitted to this broad active region, which is likely composed of several generators. Given the observed precision of EEG ASSR source analysis, we conclude EEG is an appropriate method for studying hemispheric lateralisation effects. 


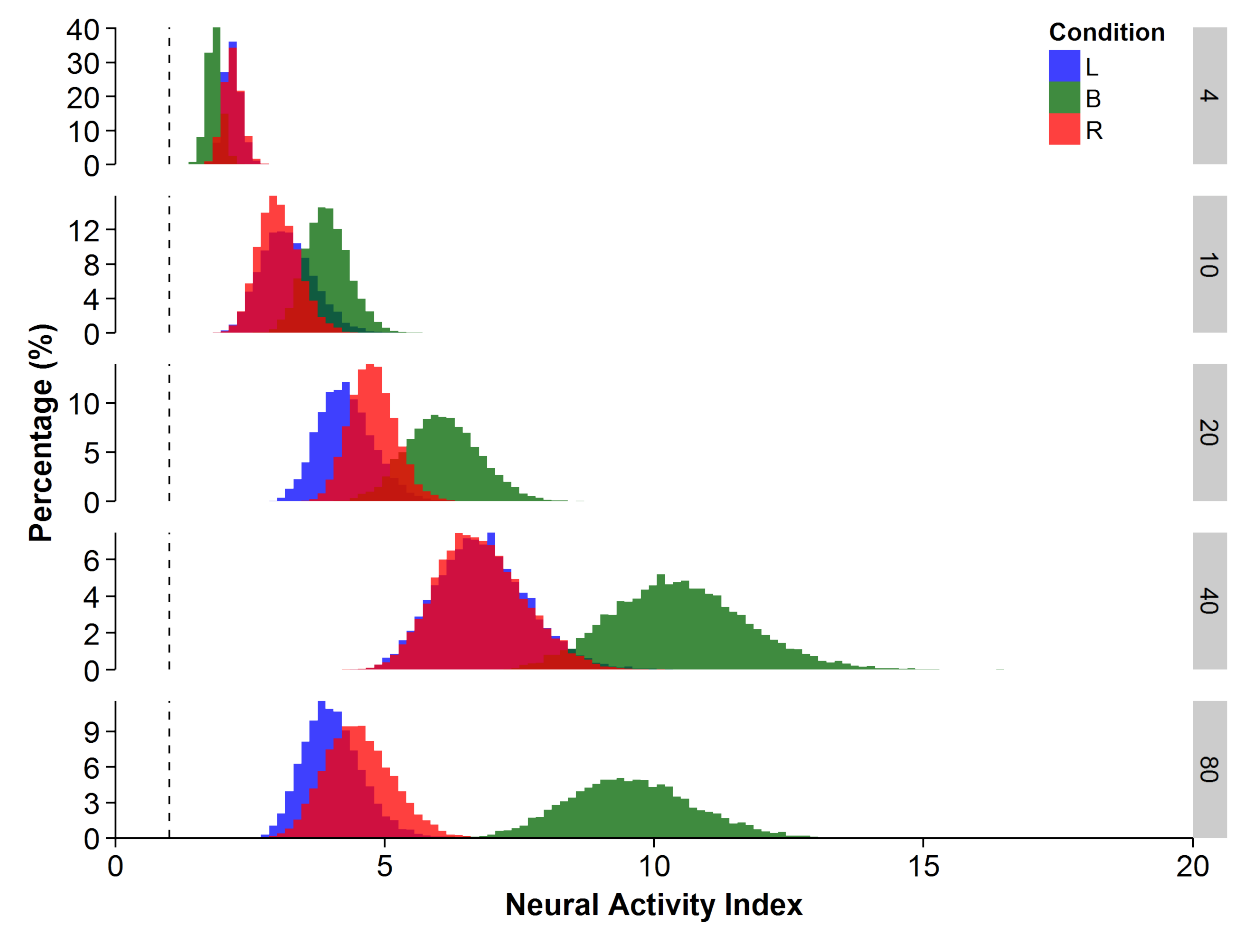

Figure 6: Distribution of source NAI values averaged over hemispheres. Facets indicate different modulation rates and color represents stimulus modality. The vertical axis represent the percentage of occurrences from the bootstrapping procedure with 10000 resamples. The vertical line indicates a NAI of 1 , where the stimulus and control condition have equal activity.

Figure 6 summarises the NAI for each modulation rate and stimulation modality. In this figure the activity was averaged over the left and right dipoles, except for $80 \mathrm{~Hz}$ were a single source was identified. Bilateral stimulation was found to have a constructive effect on the NAI for stimulation at 80, 40, 20 and $10 \mathrm{~Hz}$, and an inhibiting effect for $4 \mathrm{~Hz}$. The bilateral effect has previously been observed on the scalp level for 4 and $80 \mathrm{~Hz}$ (Poelmans et al. 2012b), however they reported no effect for $20 \mathrm{~Hz}$. These results indicate that the increase in activity during bilateral stimulation may not be caused by additional brainstem processing, but by increased cortical activity.

Figure 7 summarises the hemispheric contribution for each modulation rate and side of stimulation. Table 1 summarises the percentage of bootstrap 


\begin{tabular}{lccc}
\hline & Left & Bilateral & Right \\
\hline $4 \mathrm{~Hz}$ & 73 & 79 & 7 \\
$10 \mathrm{~Hz}$ & $3^{*}$ & $3^{*}$ & 12 \\
$20 \mathrm{~Hz}$ & 24 & 41 & 83 \\
$40 \mathrm{~Hz}$ & $99^{*}$ & 91 & $98^{*}$ \\
\hline
\end{tabular}

Table 1: Percentage of bootstrap resamples with a LI greater than 0, indicating a right hemispheric preference. A star indicates over $95 \%$ of resamples were lateralised left or right.

samples determined to be right lateralised. Estimates were not obtained for the $80 \mathrm{~Hz}$ condition as only a single source was extracted; however, significant scalp level laterality has been shown for $80 \mathrm{~Hz}$ modulation rates (Poelmans et al. 2012b, Vanvooren et al.|2014, 2015). A right hemisphere preference was observed for $40 \mathrm{~Hz}$ modulation rates regardless of the stimulation mode, this result is consistent with previous MEG observations (Roß et al. 2005). Contralateral activation was observed for the $4 \mathrm{~Hz}$ modulation rate for monaural stimulation, consistent with MRI observations (Langers et al. 2005) but contradictory to scalp level EEG results (Poelmans et al. 2012b). A $4 \mathrm{~Hz}$ right hemispheric preference was observed for bilateral stimulation, consistent with MEG observations (Hymers et al. 2010, Simpson et al. 2012). The $10 \mathrm{~Hz}$ modulation rate preferentially activated the left hemisphere. Whereas, 20 $\mathrm{Hz}$ activated the ipsilateral hemisphere for monaural stimulation, consistent with scalp level EEG observations (Poelmans et al. 2012b). These results are generally consistent with previous findings across imaging techniques, illustrating EEG source analysis provides the sensitivity required to study hemispheric lateralisation effects. This result indicates that even though the neural processing locations are similar for different modulation rates (Giraud et al. 2000), underlying functional networks may differ across speech envelope features. By systematically varying modulation rate and stimulation mode within a single cohort, we have shown that large LI differences can be found for closely spaced low frequency AM stimuli. This highlights that particular attention should be paid to both modulation rate and presentation ear when studying envelope processing. 


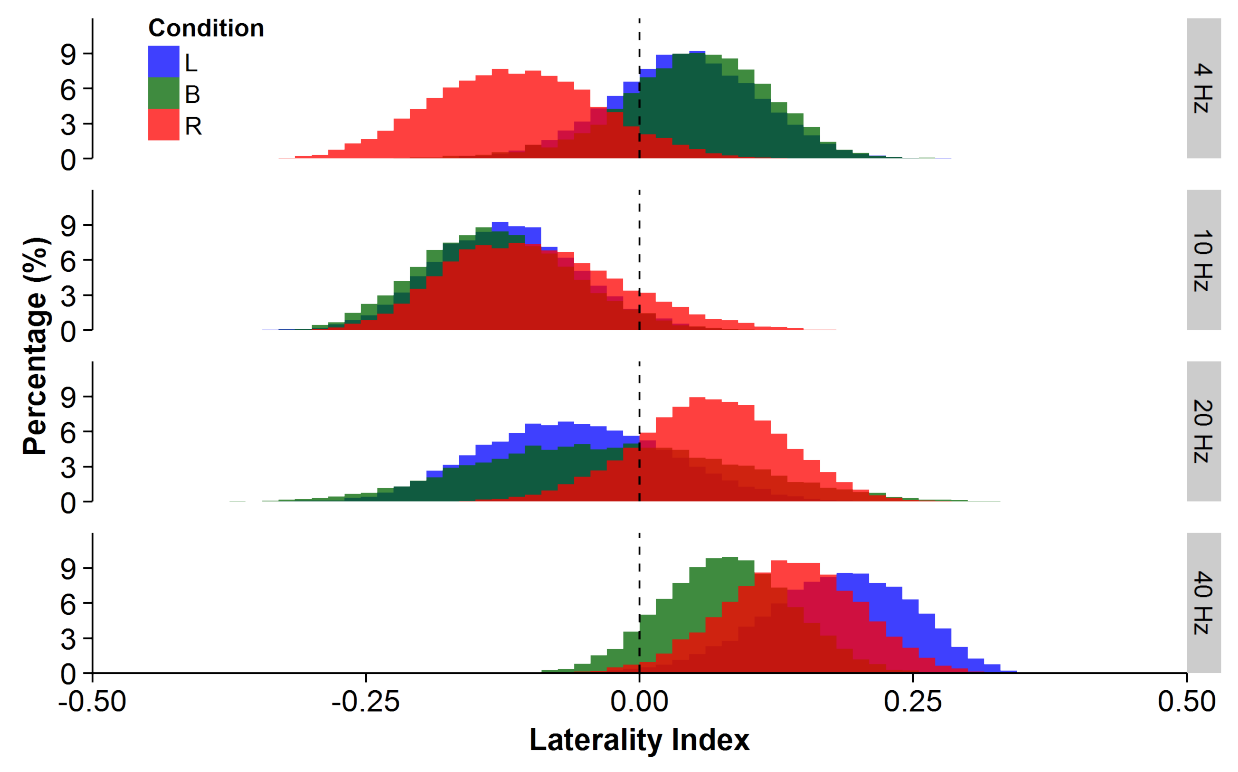

Figure 7: Distribution of source laterality values for different modulation rates and stimulation conditions drawn a bootstrapping procedure with 10000 resamples. Facets represent modulation rate, color represents stimulus modality. 


\subsection{Electrical Analysis}

The CI cohort comprised of five listeners implanted in the right ear. $50 \mathrm{~min}$ of data was measured for each of the three CI electrode pairs, totalling $150 \mathrm{~min}$ of measurements, enough data to obtain accurate source estimates [section 3.1.1. EEG recorded during $\mathrm{CI}$ stimulation contains artifacts that must be removed prior to further signal processing. Due to the continuous nature of the steady state response, removal of artifacts is particularly difficult. EASSRs were consistently measured by Hofmann and Wouters (2010) using a blanking method, later by Luke and Wouters (2016) using a Kalman filter, and Deprez et al. (2014) using ICA. The blanking method of CI artifact removal is demonstrated in figure 1. As a sanity check, the beamforming technique was also applied to recordings without blanking, this resulted in a single extremely large point source at the location of the CI. The effect of deafness and sensitive periods has been investigated using transient cortical and transient evoked potentials on scalp and source level (Gordon et al. 2013, Kral and Sharma 2012, Gilley et al. 2008, Sharma et al. 2009). However, EASSRs have particular perceptual relevance due to the similarity with CI envelope coding strategies, clinical pulse rates, and relations with prominent oscillations in the speech envelope.

\subsubsection{Description Of Neural Activity}

Figure 8a shows the NAI estimate for the electrical hearing group, dipole locations are illustrated for apical, middle and basal CI electrode conditions, as well as all data combined. The remainder of results are for the combined data. A source was consistently identified in the left hemisphere with NAI similar to the $40 \mathrm{~Hz}$ acoustic hearing population. No clear right source was identified, for laterality calculations a right source was placed in the mirror location to the left source. The coordinates of the $40 \mathrm{~Hz}$ left sources were ($39,-13,10) \mathrm{mm}$ in the standard Talairach coordinate system, the coordinates of the mirrored right source were $(39,13,10) \mathrm{mm}$. To ensure the additional signal processing steps did not affect the $\mathrm{NAI}$ or $\mathrm{LI}$ measures, the acoustic hearing group was reprocessed with exactly the same processing as the CI cohort. Figure $8 \mathrm{~b}$ shows the estimate the for normal hearing cohort with blanking and electrode removal. The location and lateralisation are similar to section 3.1. however the estimate has a lower resolution due to the reduced temporal and spatial sampling. 

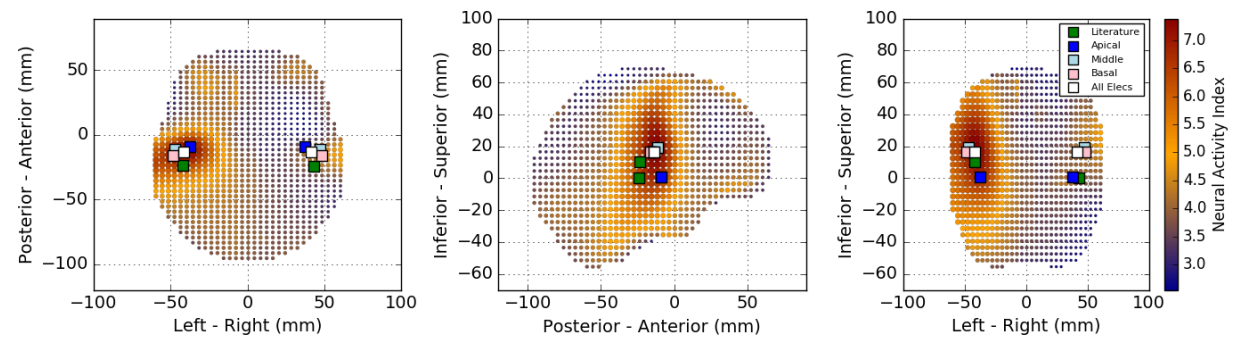

(a) Cochlear Implant
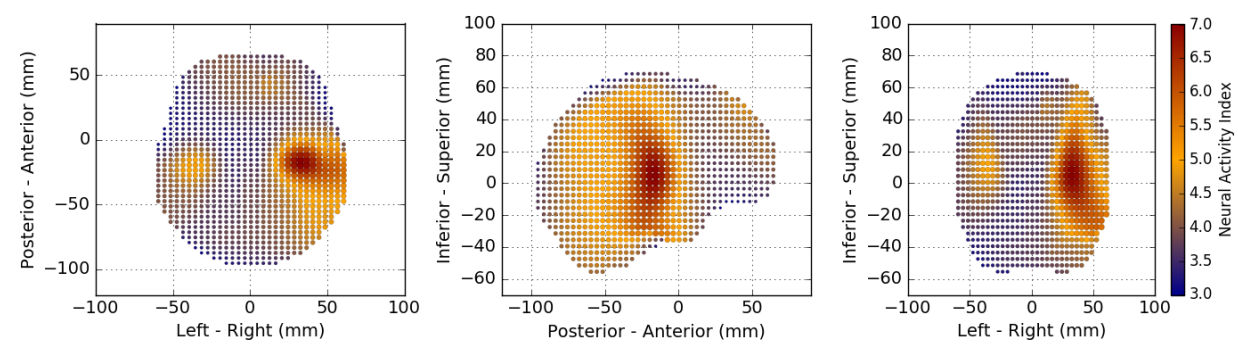

(b) Acoustic Hearing

Figure 8: $40 \mathrm{~Hz}$ ASSR NAI estimates for $\mathrm{CI}$ and acoustic listeners with identical preprocessing. CI users are implanted on the right ear, for the acoustic hearing group stimuli are presented to the right ear. Dipoles are shown for apical, middle and basal CI electrodes, and for all data combined. Green markers indicate the acoustic hearing literature reference locations. 


\subsection{Comparison Of Groups}

The CI cohort laterality index was -0.32 , with jackknife standard deviation of 0.11 , this is illustrated by the vertical line and shading in figure 9 . The reprocessed acoustic hearing cohort laterality index was 0.19 (SD 0.09), illustrated as a distribution in figure 9. The probability the CI LI was drawn from the acoustic cohort distribution was $<1 \%$.

This result contributes to understanding how unilateral implantation affects the auditory pathway after a period of auditory deprivation. Minimising the period of auditory deprivation prior to implantation improves speech perception (Boons et al. 2012, Lazard et al. 2012), but the neural mechanisms underlying this relation are still under investigation (Kral and Sharma 2012). Hemispheric asymmetry of auditory processing has been studied in humans with typical hearing (Schonwiesner et al. 2007) and unilateral deafness. Unilateral deafness does not affect the lateralisation of activity at the brainstem level (Langers et al. 2005). Changes to cortical lateralisation have been observed for people with unilateral deafness. Ponton et al. (2001) demonstrated a difference in long-latency auditory evoked potentials between normal hearing and unilateral deaf listeners, they suggested this difference was caused by changes in the generators producing the $\mathrm{N}_{1}$ potential. Additionally, source modelling of long-latency responses has shown reduced hemispheric asymmetry for listeners with unilateral deafness relative to normal hearing controls, particularly for participants with left ear unilateral deafness (Khosla et al. 2003). Burton et al. (2012) further demonstrated the effect of ear on hemispheric asymmetry in unilaterally deaf listeners. This study investigated a group of right implanted unilateral CI users. Given the observed effect of ear in studies of unilateral deafness, future CI studies of hemispheric asymmetry should consider side of implantation as a factor in their analysis. Hemispheric asymmetry has also been investigated for cochlear implant users. Gilley et al. (2008) illustrated children implanted after seven years develop reorganised cortical pathways, as the source location of the CI group was different to the normal hearing controls. This was not observed in our data, possibly as all participants became deaf postlingually. Gordon et al. (2013) used EEG to analyse the neural generators of the low rate $\mathrm{P} 1$ complex and observed increased contralateral activity for users with more than 1.5 years unilateral use. However, CIs encode the speech envelope using high rate modulated pulse trains, the EASSR stimulus is designed to be similar to clinical stimuli. Our results complement Gordon et al. (2013), preferential contralateral activation for both transient and sustained neural 


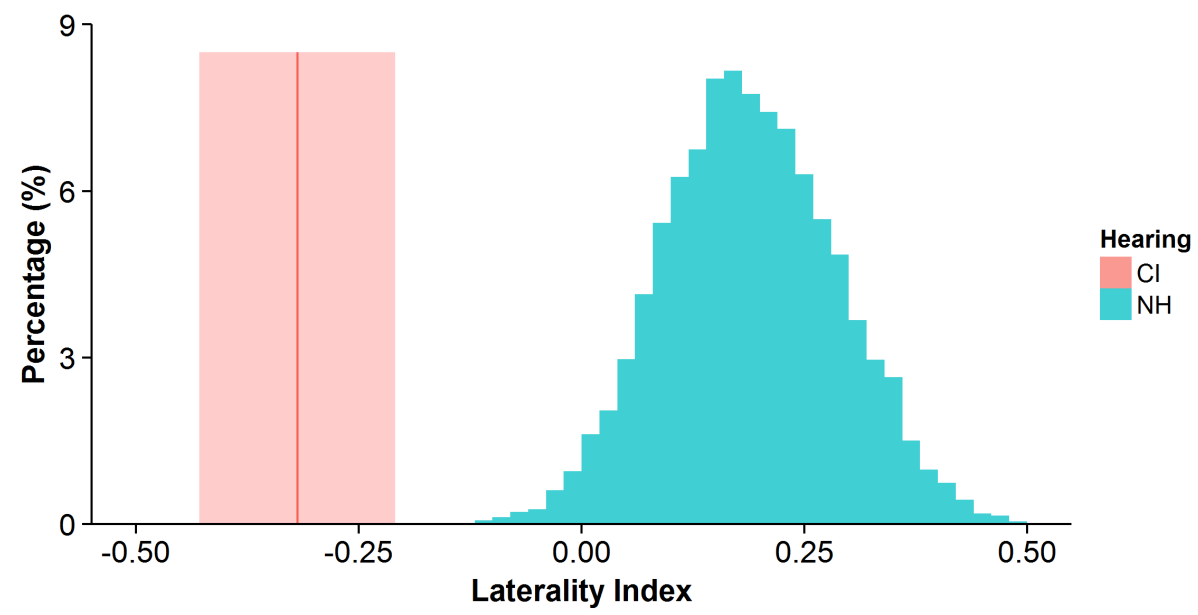

Figure 9: Comparison of $40 \mathrm{~Hz}$ laterality between acoustic hearing and CI group. The distribution of occurrences is shown for the acoustic hearing population, determined via bootstrapping with 10000 resamples. The vertical line represents the mean electrical hearing laterality, the shading represents the jackknife estimate standard deviation.

evoked responses indicates that the auditory pathway is altered in unilateral CI users to favour the contralateral side relative to acoustic listeners.

\section{Conclusion}

This study investigated if unilateral CI users process speech envelope like stimuli similarly to the acoustic hearing population. A method of source analysis was presented and the accuracy and precision were investigated. The neural generators of AM ASSRs where characterised in an acoustic hearing cohort, locations and lateralisation matched and extended findings from other tomography's.

A novel beamforming extension was introduced which facilitated source analysis of EASSRs. The neural generators of $40 \mathrm{~Hz}$ EASSRs were determined for a group of five CI patients. Results indicated exaggerated contralateral activation for $\mathrm{CI}$ users relative to the acoustic hearing population, complementing results from transient stimuli studies. This is the first study characterising the location and hemispheric laterality of neural generators of ASSRs to low-rate speech envelope modulations using monaural and bilateral stimuli. 


\section{Acknowledgment}

The work leading to this deliverable and the results described therein has received funding from the People Programme (Marie Curie Actions) of the European Unions Seventh Framework Programme FP7/2007-2013/ under REA grant agreement PITN-GA-2012-317521.

Support was also provided by research grant (G.066213) of the Research Foundation Flanders (FWO), and a research grant (110722) by the Flemish Agency for Innovation by Science and Technology (IWT).

\section{References}

Boons, T., Brokx, J. P., Dhooge, I., Frijns, J. H., Peeraer, L., Vermeulen, A., Wouters, J., and Van Wieringen, A. (2012). Predictors of spoken language development following pediatric cochlear implantation. Ear and hearing, 33(5):617-639.

Burton, H., Firszt, J. B., Holden, T., Agato, A., and Uchanski, R. M. (2012). Activation lateralization in human core, belt, and parabelt auditory fields with unilateral deafness compared to normal hearing. Brain research, 1454:33-47.

Cho, J.-H., Vorwerk, J., Wolters, C. H., and Knösche, T. R. (2015). Influence of the head model on eeg and meg source connectivity analyses. NeuroImage, 110:60-77.

Cuffin, B. N., Schomer, D. L., Ives, J. R., and Blume, H. (2001). Experimental tests of eeg source localization accuracy in realistically shaped head models. Clinical neurophysiology, 112(12):2288-2292.

Dalal, S. S., Sekihara, K., and Nagarajan, S. S. (2006). Modified beamformers for coherent source region suppression. IEEE Transactions on Biomedical Engineering, 53(7):1357-1363.

Deprez, H., Gransier, R., Hofmann, M., van Wieringen, A., Wouters, J., and Moonen, M. (2017). Characterization of cochlear implant artifacts in electrically evoked auditory steady-state responses. Biomedical Signal Processing and Control, 31:127-138.

Deprez, H., Hofmann, M., Van Wieringen, A., Wouters, J., and Moonen, M. (2014). Cochlear implant artifact rejection in electrically evoked audi- 
tory steady state responses. In IEEE Proceedings of the European Signal Processing Conference, number 22, pages 1995-1999.

Dimitrijevic, A., John, M. S., and Picton, T. W. (2004). Auditory steadystate responses and word recognition scores in normal-hearing and hearingimpaired adults. Ear and Hearing, 25(1):68-84.

Edwards, E. and Chang, E. F. (2013). Syllabic $(2-5 \mathrm{~Hz})$ and fluctuation $(1-10 \mathrm{~Hz})$ ranges in speech and auditory processing. Hearing Research, 305:113-134.

Efron, B. (1979). Bootstrap methods: another look at the jackknife. The Annals of Statistics, 7(1):1-26.

Friesen, L. M., Shannon, R. V., Baskent, D., and Wang, X. (2001). Speech recognition in noise as a function of the number of spectral channels: comparison of acoustic hearing and cochlear implants. The Journal of the Acoustical Society of America, 110(2):1150-1163.

Fu, Q. (2002). Temporal processing and speech recognition in cochlear implant users. Neuroreport, 13:1635-1639.

Gilley, P. M., Sharma, A., and Dorman, M. F. (2008). Cortical reorganization in children with cochlear implants. Brain research, 1239:56-65.

Giraud, A.-L., Lorenzi, C., Ashburner, J., Wable, J., Johnsrude, I., Frackowiak, R., and Kleinschmidt, A. (2000). Representation of the temporal envelope of sounds in the human brain. Journal of Neurophysiology, 84(3):1588-1598.

Gordon, K. A., Wong, D., and Papsin, B. C. (2013). Bilateral input protects the cortex from unilaterally-driven reorganization in children who are deaf. Brain, awt052:1 - 17.

Gramfort, A., Luessi, M., Larson, E., Engemann, D. A., Strohmeier, D., Brodbeck, C., Goj, R., Jas, M., Brooks, T., Parkkonen, L., and Hämäläinen, M. (2013). Meg and eeg data analysis with mne-python. Frontiers in Neuroscience, $7(267)$.

Gramfort, A., Luessi, M., Larson, E., Engemann, D. A., Strohmeier, D., Brodbeck, C., Parkkonen, L., and Hämäläinen, M. S. (2014). Mne software for processing meg and eeg data. Neuroimage, 86:446-460. 
Gransier, R., Deprez, H., Hofmann, M., Moonen, M., van Wieringen, A., and Wouters, J. (2016). Auditory steady-state responses in cochlear implant users: Effect of modulation frequency and stimulation artifacts. Hearing Research, 335:149-160.

Greenberg, S., Hollenback, J., and Ellis, D. (1996). Insights into spoken language gleaned from phonetic transcription of the switchboard corpus. In International Conference on Spoken Language Processing, pages 32-35.

Greenberg, S. and Takayuki, A. (2004). What are the essential cues for understanding spoken language? IEICE transactions on information and systems, 87(5):1059-1070.

Groß, J., Kujala, J., Hämäläinen, M., Timmermann, L., Schnitzler, A., and Salmelin, R. (2001). Dynamic imaging of coherent sources: studying neural interactions in the human brain. Proceedings of the National Academy of Sciences, 98(2):694-699.

Herdman, A. T., Lins, O., Van Roon, P., Stapells, D. R., Scherg, M., and Picton, T. W. (2002). Intracerebral sources of human auditory steadystate responses. Brain topography, 15(2):69-86.

Hofmann, M. and Wouters, J. (2010). Electrically evoked auditory steady state responses in cochlear implant users. Journal of the Association for Research in Otolaryngology, 11(2):267 - 82.

Hofmann, M. and Wouters, J. (2012). Improved electrically evoked auditory steady-state response thresholds in humans. Journal of the Association for Research in Otolaryngology, 13(4):573-589.

Hymers, M., Prendergast, G., Johnson, S. R., and Green, G. G. (2010). Source stability index: A novel beamforming based localisation metric. Neuroimage, 49(2):1385-1397.

Khosla, D., Ponton, C. W., Eggermont, J. J., Kwong, B., Don, M., and Vasama, J.-P. (2003). Differential ear effects of profound unilateral deafness on the adult human central auditory system. Journal of the Association for Research in Otolaryngology, 4(2):235-49.

Kral, A. and Sharma, A. (2012). Developmental neuroplasticity after cochlear implantation. Trends in neurosciences, 35(2):111-122. 
Langers, D. R., van Dijk, P., and Backes, W. H. (2005). Lateralization, connectivity and plasticity in the human central auditory system. Neuroimage, 28(2):490-499.

Lazard, D., Giraud, A., Truy, E., and Lee, H. (2011). Evolution of nonspeech sound memory in postlingual deafness: implications for cochlear implant rehabilitation. Neuropsychologia, 49(9):2475-2482.

Lazard, D. S., Vincent, C., Venail, F., Van de Heyning, P., Truy, E., Sterkers, O., Skarzynski, P. H., Skarzynski, H., Schauwers, K., O’Leary, S., et al. (2012). Pre-, per- and postoperative factors affecting performance of postlinguistically deaf adults using cochlear implants: a new conceptual model over time. PloS one, 7(11):e48739.

Lemire, D. (2006). Streaming maximum-minimum filter using no more than three comparisons per element. Nordic Journal of Computing, 13(4):328339 .

Lucka, F., Pursiainen, S., Burger, M., and Wolters, C. H. (2012). Hierarchical bayesian inference for the eeg inverse problem using realistic fe head models: depth localization and source separation for focal primary currents. Neuroimage, 61(4):1364-1382.

Luke, R., Van Deun, L., Hofmann, M., Van Wieringen, A., and Wouters, J. (2015). Assessing temporal modulation sensitivity using electrically evoked auditory steady state responses. Hearing Research, 324:37-45.

Luke, R. and Wouters, J. (2016). Kalman filter based estimation of auditory steady state response parameters. IEEE Transactions on Neural Systems and Rehabilitation Engineering, in press.

Majdani, O., Rau, T. S., Götz, F., Zimmerling, M., Lenarz, M., Lenarz, T., Labadie, R., and Leinung, M. (2009). Artifacts caused by cochlear implants with non-removable magnets in 3t mri: phantom and cadaveric studies. European Archives of Oto-Rhino-Laryngology, 266(12):1885-1890.

Picton, T. W., John, M. S., Dimitrijevic, A., and Purcell, D. (2003). Human auditory steady-state responses. International Journal of Audiology, 42(4):177-219.

Poelmans, H., Luts, H., Vandermosten, M., Boets, B., Ghesquière, P., and Wouters, J. (2012a). Auditory steady state cortical responses indicate deviant phonemic-rate processing in adults with dyslexia. Ear and Hearing, 33(1):134-143. 
Poelmans, H., Luts, H., Vandermosten, M., Ghesquière, P., and Wouters, J. (2012b). Hemispheric asymmetry of auditory steady-state responses to monaural and diotic stimulation. Journal of the Association for Research in Otolaryngology, 13(6):867-876.

Ponton, C. W., Vasama, J. P., Tremblay, K., Khosla, D., Kwong, B., and Don, M. (2001). Plasticity in the adult human central auditory system: evidence from late-onset profound unilateral deafness. Hearing research, 154(1-2):32-44.

Prendergast, G., Johnson, S. R., and Green, G. G. (2010). Temporal dynamics of sinusoidal and non-sinusoidal amplitude modulation. European Journal of Neuroscience, 32(9):1599-1607.

Rance, G. (2008). Auditory Steady-State Response Generation, Recording, and Clinical Application. Plural Publishing Inc.

Rance, G., Rickards, F. W., Cohen, L. T., De Vidi, S., and Clark, G. M. (1995). The automated prediction of hearing thresholds in sleeping subjects using auditory steady-state evoked potentials. Ear and Hearing, 16(5):499-507.

Reyes, S. A., Lockwood, A. H., Salvi, R. J., Coad, M. L., Wack, D. S., and Burkard, R. F. (2005). Mapping the 40-hz auditory steady-state response using current density reconstructions. Hearing Research, 204(1):1-15.

Reyes, S. A., Salvi, R. J., Burkard, R. F., Coad, M. L., Wack, D. S., Galantowicz, P. J., and Lockwood, A. H. (2004). Pet imaging of the $40 \mathrm{hz}$ auditory steady state response. Hearing Research, 194(1):73-80.

Rosen, S. (1992). Temporal information in speech: acoustic, auditory and linguistic aspects. Philosophical Transactions of the Royal Society of London. Series B: Biological Sciences, 336(1278):367-373.

Roß, B., Borgmann, C., Draganova, R., Roberts, L. E., and Pantev, C. (2000). A high-precision magnetoencephalographic study of human auditory steady-state responses to amplitude-modulated tones. The Journal of the Acoustical Society of America, 108(2):679-691.

Roß, B., Herdman, A., and Pantev, C. (2005). Right hemispheric laterality of human $40 \mathrm{hz}$ auditory steady-state responses. Cerebral Cortex, 15(12):2029-2039. 
Roß, B., Picton, T., Herdman, A., and Pantev, C. (2003). The effect of attention on the auditory steady-state response. Neurology clinical neurophysiology, 2004:22-22.

Schonwiesner, M., Krumbholz, K., Rubsamen, R., Fink, G. R., and von Cramon, D. Y. (2007). Hemispheric asymmetry for auditory processing in the human auditory brain stem, thalamus, and cortex. Cerebral Cortex, $17(2): 492-9$.

Sekihara, K., Nagarajan, S. S., Poeppel, D., Marantz, A., and Miyashita, Y. (2001). Reconstructing spatio-temporal activities of neural sources using an meg vector beamformer technique. IEEE Transactions on Biomedical Engineering, 48(7):760-771.

Shannon, R. V., Zeng, F.-G., Kamath, V., Wygonski, J., and Ekelid, M. (1995). Speech recognition with primarily temporal cues. Science, 270(5234):303-304.

Sharma, A., Nash, A. A., and Dorman, M. (2009). Cortical development, plasticity and re-organization in children with cochlear implants. Journal of communication disorders, 42(4):272-9.

Simpson, M. I., Woods, W. P., Prendergast, G., Johnson, S. R., and Green, G. G. (2012). Stimulus variability affects the amplitude of the auditory steady-state response. PloS one, 7(4):e34668.

Steinmann, I. and Gutschalk, A. (2011). Potential fmri correlates of 40-hz phase locking in primary auditory cortex, thalamus and midbrain. Neuroimage, 54(1):495-504.

Teale, P., Collins, D., Maharajh, K., Rojas, D. C., Kronberg, E., and Reite, M. (2008). Cortical source estimates of gamma band amplitude and phase are different in schizophrenia. Neuroimage, 42(4):1481-1489.

Tukey, J. W. (1958). Bias and confidence in not-quite large samples. In Annals of Mathematical statistics, volume 29, pages 614-614.

Van Veen, B. D. and Buckley, K. M. (1988). Beamforming: A versatile approach to spatial filtering. IEEE ASSP magazine, 5(2):4-24.

Van Veen, B. D., van Drongelen, W., Yuchtman, M., and Suzuki, A. (1997). Localization of brain electrical activity via linearly constrained minimum variance spatial filtering. Biomedical Engineering, IEEE Transactions on, 44(9):867-880. 
Van Wieringen, A. and Wouters, J. (2008). List and lint: Sentences and numbers for quantifying speech understanding in severely impaired listeners for flanders and the netherlands. International journal of audiology, $47(6): 348-355$.

Vanvooren, S., Hofmann, M., Poelmans, H., Ghesquière, P., and Wouters, J. (2015). Theta, beta and gamma rate modulations in the developing auditory system. Hearing research, 327:153-162.

Vanvooren, S., Poelmans, H., Hofmann, M., Ghesquiére, P., and Wouters, J. (2014). Hemispheric asymmetry in auditory processing of speech envelope modulations in prereading children. The Journal of Neuroscience, 34(4):1523-1529.

Wong, D. and Gordon, K. A. (2009). Beamformer suppression of cochlear implant artifacts in an electroencephalography dataset. IEEE Transactions on Biomedical Engineering, 56(12):2851-2857.

Wouters, J., McDermott, H. J., and Francart, T. (2015). Sound coding in cochlear implants: from electric pulses to hearing. IEEE Signal Processing Magazine, 32(2):67-80. 